\title{
ADAM9 expression in pancreatic cancer is associated with tumour type and is a prognostic factor in ductal adenocarcinoma
}

\author{
R Grützmann', J Lüttges², B Sipos², O Ammerpohl', F Dobrowolski', I Alldinger', S Kersting', D Ockert', \\ R Koch ${ }^{4}$, H Kalthoff ${ }^{3}$, HK Schackert ${ }^{5}$, HD Saeger', G Klöppel ${ }^{2}$ and C Pilarsky, \\ 'Department of Visceral, Thoracic and Vascular Surgery, University Hospital Carl Gustav Carus, Technical University of Dresden, Fetscherstrasse 74, \\ Dresden 01 307, Germany; ${ }^{2}$ Department of Pathology, University of Kiel, Germany; ${ }^{3}$ Molecular Oncology Research Group, Clinic for General Surgery and \\ Thoracic Surgery, University of Kiel, Germany; ${ }^{4}$ Institute of Medical Informatics and Biometrics, Technical University of Dresden, Germany; and \\ ${ }^{5}$ Department of Surgical Research, University Hospital Carl Gustav Carus, Technical University of Dresden, Germany
}

\begin{abstract}
Gene expression profiling revealed ADAM9 to be distinctly overexpressed in pancreatic ductal adenocarcinoma (PDAC). We examined the relevance of ADAM9 expression in PDAC diagnosis and prognosis. A total of 59 infiltrating PDACs, 32 specimens from patients with chronic pancreatitis, II endocrine tumours and 24 acinar cell carcinomas were immunohistochemically analysed for ADAM9 expression. Staining for ADAM9 was detected in 58 out of 59 (98.3\%) PDACs and in two out of 24 (8.3\%) acinar cell carcinomas, but not in endocrine tumours. In the non-neoplastic pancreas, whether normal or chronically inflamed, ADAM9 was expressed in centroacinar and intralobular duct cells, but not in interlobular duct cells and their hyperplastic lesions. Pancreatic ductal adenocarcinomas showing cytoplasmic ADAM9 expression correlated with poor tumour differentiation and also with shorter overall survival than in cases showing only an apical membranous staining pattern $(P=0.00 \mathrm{I})$. Multivariate analysis identified cytoplasmic ADAM9 expression as an independent marker of shortened survival in a set of 42 curatively (R0) resected PDAC ( $P<0.05$, hazard ratio $2.85,95 \%$ confidence interval: $\mid .21-6.71)$. The results show that ADAM9 expression distinguishes PDACs from other solid pancreatic tumours. In addition, cytoplasmic ADAM9 overexpression is associated with poor differentiation and shortened survival. Therefore, ADAM9 overexpression might contribute to the aggressiveness of PDACs.

British Journal of Cancer (2004) 90, 1053- 1058. doi:I0.1038/sj.bjc.6601645 www.bjcancer.com

(c) 2004 Cancer Research UK
\end{abstract}

Keywords: pancreatic cancer; ADAM9; gene profiling; immunohistochemistry; prognosis; survival

Pancreatic ductal adenocarcinoma (PDAC) is an important cause of malignancy-related death. In the US it ranks fifth among the leading causes of cancer death, accounting for approximately 30000 deaths annually (Jemal et al, 2003). Apart from surgery, there is no effective therapy and even resected patients frequently die within 1 year of the operation. In the past years, several genes have been identified as related to the development of PDAC (Hruban et al, 1998; Slebos et al, 2000). However, considering the complexity of the genome, it is most likely that most of the molecular changes causing PDAC still need to be elucidated (Bardeesy and DePinho, 2002). Moreover, there is still a need for prognostic markers in this devastating cancer disease.

Recently, others and we identified ADAM9 as one of the genes that is overexpressed in PDAC, when compared to normal pancreatic tissue using DNA microarray transcript profiling. This result was validated by an RT - PCR analysis in PDAC cell lines, which revealed ADAM9 expression in 13 of the 20 cell lines, and by immunohistochemistry in a small set of PDACs (Grutzmann et al, 2003; Iacobuzio-Donahue et al, 2003). ADAM9 overexpression was also demonstrated in prostate, breast and liver cell carcinomas (McCulloch et al, 2000; Le Pabic et al, 2003; O'Shea et al, 2003).

\footnotetext{
*Correspondence: Dr C Pilarsky;
}

E-mail: Christian.Pilarsky@mailbox.tu-dresden.de

Received 29 August 2003; revised 26 November 2003; accepted I5 December 2003
ADAM9 is a member of the large ADAM family of proteases, which are type I transmembrane proteins with both metalloproteinase and disintegrin-containing extracellular domains. The ADAMs are implicated in the proteolytic processing of membrane-bound TNF $\alpha$ precursors and are involved in modulating cell-cell and cellmatrix interactions (Amour et al, 2002).

Although at present the precise molecular and biological mechanisms of ADAM9 remain to be elucidated, ADAM9 may be involved in the carcinogenesis of PDAC. For this reason, we were interested in determining whether the proposed differential expression of ADAM9 in PDAC at the RNA level could be confirmed at the protein level as well. Moreover, if possible, we wanted to evaluate the prognostic significance of ADAM9 expression in PDACs. In this study, we demonstrate that ADAM9 expression distinguishes PDACs from pancreatic acinar cell carcinomas and endocrine tumours. In addition, the cytoplasmic expression of ADAM9 has a prognostic potential.

\section{MATERIAL AND METHODS}

\section{Patients and tissues}

Formalin-fixed, paraffin-embedded tissue blocks were obtained from surgical specimens from 59 patients (mean age 59 years; range $31-76$ ) with PDAC, who were operated at the Department of Visceral, Thoracic and Vascular Surgery, University Hospital Carl 
Gustav Carus, Technical University of Dresden, between 1996 and 2001. All PDAC patients received standard surgical therapy based on their clinical stages. In addition, tissue samples were obtained from surgical specimens from 32 patients with chronic pancreatitis, 11 patients with pancreatic endocrine tumours and 24 patients with acinar cell carcinomas. These tissues were selected from the institutional files and consultation files of the Department of Pathology, University of Kiel. Five patients with pancreatic endocrine tumours, one patient with acinar cell carcinoma and all 32 patients with chronic pancreatitis were operated at the Department of General Surgery and Thoracic Surgery, University of Kiel, between 1994 and 2002. All patients were randomly selected without stratification for known preoperative or pathological prognostic factors. The PDACs were staged (TNM classification) and reclassified histologically (JL, GK) according to the WHO classification (Kloppel et al, 2000). The clinicopathological features of the PDACs are listed in Table 1.

\section{Immunohistochemistry}

Sections $(4 \mu \mathrm{m})$ were cut from formalin-fixed, paraffin-embedded pancreatic tissue. The sections were mounted on superfrost slides (Menzel Gläser, Braunschweig, Germany), dewaxed with xylene and were gradually hydrated. Antigen was exposed by heating the sections under high pressure in Tris-EDTAcitrate buffer for $3 \mathrm{~min}$. The primary goat polyclonal anti-mouse ADAM9 antibody (AF949, R\&D Systems, Wiesbaden, Germany), which crossreacts with human ADAM9, was diluted $\left(15 \mu \mathrm{g} \mathrm{ml}^{-1}\right)$ in PBS containing $2 \%$ horse serum (Vector Laboratories, Burlingame, CA, USA). After incubation for $45 \mathrm{~min}$, the reaction was detected with a biotinylated anti-goat antibody $\left(5 \mu \mathrm{g} \mathrm{ml}^{-1}\right.$, Vector Laboratories) and avidin-biotin-peroxidase (ABC ELITE, Vector Laboratories). Diaminobenzidine served as chromogen. Afterwards, the slides were briefly counterstained with haematoxylin. For the negative control, the primary antibody was omitted. The staining intensity was evaluated semiquantitatively as negative, weak, moderate or strong. The final results, however, were recorded as positive (weak, moderate or strong staining) or negative staining only. In addition, the staining pattern was evaluated and a distinction was made between labelling of the luminal (apical) cell membrane of tumour cells forming glandular structures, the basolateral cell membrane and the cytoplasm. The staining was evaluated independently by two pathologists, who were unaware of patient survival.

\section{Statistical analysis}

The Mantel-Haenszel test was used to assess the correlation between the clinicopathological findings and ADAM9 expression. For the survival analysis, a univariate analysis of 59 patients with PDAC was performed using the Kaplan-Meier method, MantelHaenszel estimation of the hazard ratios and log-rank tests for comparing between the strata (Peto et al, 1977). The histological grade, pTNM stage, $\mathrm{R}$ (residual tumour) stage and UICC stage were evaluated in a multivariate analysis using the Cox proportional regression hazard model. The differences at $P<0.05$ were considered significant. For statistical evaluation, the SAS/STAT software version 8 and SPSS software v. 11.0.1 were used.

\section{RESULTS}

\section{ADAM9 immunostaining}

Immunohistochemically, 58 of the 59 PDACs were positive for ADAM9 (Table 1, Figure 1A). In 57 of the 59 PDACs, there was staining of the luminal cell membrane in areas with glandular

Table I ADAM9 expression patterns in pancreatic ductal adenocarcinomas (PDAC) correlated with clinicopathological variables

\begin{tabular}{|c|c|c|c|c|c|c|c|c|c|}
\hline & \multirow[b]{2}{*}{ Total no. of patients } & \multicolumn{2}{|c|}{ ADAM9 expression } & \multicolumn{2}{|c|}{ Luminal ADAM9 } & \multicolumn{2}{|c|}{ Basolateral ADAM9 } & \multicolumn{2}{|c|}{ Cytoplasmic ADAM9 } \\
\hline & & + & - & + & - & + & - & + & - \\
\hline PDAC & 59 & 58 & I & 57 & 2 & 32 & 27 & 17 & 42 \\
\hline \multicolumn{10}{|c|}{ Histological grade } \\
\hline $\mathrm{Gl} / 2$ & 33 & 32 & I & 32 & 1 & 9 & 18 & 4 & 29 \\
\hline G3 & 26 & 26 & 0 & 25 & 1 & 23 & 3 & 13 & 13 \\
\hline \multicolumn{10}{|c|}{ Tumour stage } \\
\hline \multicolumn{10}{|c|}{$\mathrm{pT}$} \\
\hline PTI & I & । & 0 & 1 & 0 & 0 & । & 0 & 1 \\
\hline PT2 & 6 & 6 & 0 & 6 & 0 & 4 & 2 & 2 & 4 \\
\hline pT3 & 47 & 46 & 1 & 45 & 2 & 25 & 22 & 12 & 35 \\
\hline $\mathrm{pT} 4$ & 5 & 5 & 0 & 5 & 0 & 3 & 2 & 3 & 2 \\
\hline \multicolumn{10}{|l|}{$\mathrm{pN}$} \\
\hline $\mathrm{pNO}$ & 23 & 22 & | & 21 & 2 & 12 & | | & 6 & 17 \\
\hline $\mathrm{pNI}$ & 36 & 36 & 0 & 36 & 0 & 20 & 16 & $\mid 1$ & 25 \\
\hline \multicolumn{10}{|l|}{$p M$} \\
\hline $\mathrm{pMO}$ & 52 & 51 & | & 2 & 50 & 30 & 22 & 5 & 37 \\
\hline pMI & 7 & 7 & 0 & 0 & 7 & 2 & 5 & 2 & 15 \\
\hline \multicolumn{10}{|c|}{ Residual tumour } \\
\hline RO & 42 & 41 & I & 2 & 40 & 27 & 15 & 13 & 29 \\
\hline RI & 12 & 12 & 0 & 0 & 12 & 4 & 8 & 2 & 10 \\
\hline $\mathrm{R} 2$ & 5 & 5 & 0 & 0 & 5 & 1 & 4 & 2 & 3 \\
\hline \multicolumn{10}{|c|}{ UICC stage } \\
\hline 1 & 3 & 3 & 0 & 3 & 0 & I & 2 & 2 & 2 \\
\hline 2 & 16 & 15 & I & 19 & 2 & | | & 8 & 3 & 3 \\
\hline 3 & 25 & 25 & 0 & 25 & 0 & 15 & 10 & 7 & 7 \\
\hline $4 a$ & 6 & 6 & 0 & 6 & 0 & 4 & 2 & 4 & 4 \\
\hline $4 b$ & 6 & 6 & 0 & 6 & 0 & 1 & 5 & 1 & I \\
\hline
\end{tabular}



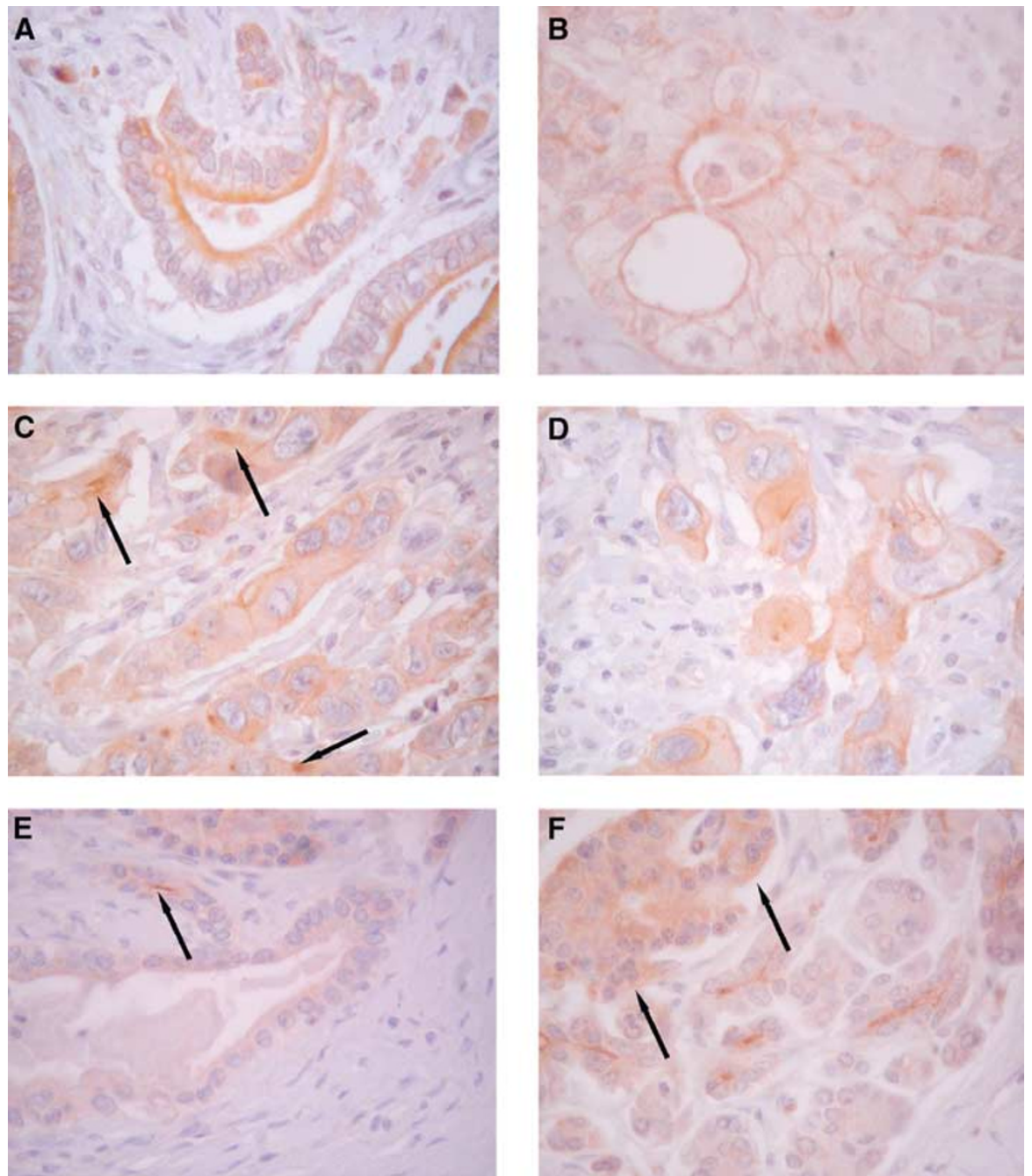

Figure I ADAM9 immunostaining in pancreatic tissues. Well-differentiated PDAC showing distinct apical ADAM9 staining at the luminal cell membrane (A); PDAC with luminal and basolateral membranous ADAM9 staining (B); poorly differentiated PDAC with basolateral membranous and focally accentuated cytoplasmic staining (arrow) (C, D); chronic pancreatitis with faint apical staining of the epithelium of a small duct (arrow) (E); normal pancreatic tissue showing staining of the luminal cell membrane of centroacinar cells and intralobular duct cells and faint granular staining of the cytoplasm of islet cells (arrow) (F).

formation. In 32 tumours, the membranous staining at the luminal side of the cell was accompanied by basolateral membrane staining. In 17 tumours, there was additionally strong cytoplasmic staining (Table 1, Figure 1B-D). Adjacent non-neoplastic pancreatic tissue as well as chronically inflamed pancreatic tissue showed weak ADAM9 expression along the luminal membrane of intralobular duct cells and centroacinar cells (Figure $1 \mathrm{E}$ and $\mathrm{F}$ ). Hyperplastic and proliferative duct lesions (i.e. pancreatic intraepithelial neoplasiaFigure 1A and 1B) were negative. Occasionally, a few acinar cells showed weak cytoplasmic staining and the islet cells generally displayed weak granular cytoplasmic labelling (Figure 1F). All endocrine tumours of the pancreas lacked cytoplasmic expression of ADAM9. This was also true of acinar cell carcinomas, with the exception of two that showed weak membranous staining in areas with dilated acinar structures, socalled glandular formation.

\section{ADAM9 expression and patient survival}

We found no significant association between cytoplasmic ADAM9 staining and patient age or stage, whereas the tumour grade was found to be statistically significant ( $P=0.03$, Fisher's exact test; Table 2). Similarly, no correlations between the intensity of ADAM9 staining, luminal ADAM9 and basolateral ADAM9 expression and these clinicopathological parameters were obtained. We also found no correlation between ADAM9 staining intensity and the occurrence of cytoplasmic ADAM9 staining (data not shown). For the univariate survival analyses, cumulative survival curves were calculated according to the Kaplan-Meier method. This analysis demonstrated statistical significance for the following parameters: tumour grade and cytoplasmic and basolateral ADAM9 expression (Figure 2, Table 3). The mean survival time of patients with PDAC without cytoplasmic ADAM9 expression was 30 months ( \pm 3 ; median $28 \pm 4$ ), compared to 16 months $( \pm 2$; median $11 \pm 1$ ) for those whose tumours showed cytoplasmic ADAM9 expression $(P<0.001$, Table 3$)$. The mean survival time for patients with PDAC who showed no basolateral ADAM9 staining was 40 months ( \pm 6 ; median $37 \pm 3$ ), compared to 18 months $( \pm 4$; median \pm 2$)$ for those PDAC patients with basolateral ADAM9 expression $(P<0.001$, Table 3$)$.

A multivariate progression analysis based on the Cox proportional hazard model was performed in order to test the 
Table 2 Relationship between cytoplasmic ADAM9 expression and various clinicopathological factors in all patients with pancreatic ductal adenocarcinomas (PDAC)

\begin{tabular}{|c|c|c|c|c|}
\hline Characteristic & All cases & Cytoplasmic ADAM9-negative & Cytoplasmic ADAM9-positive & Significance \\
\hline Age at surgery & & & & $0.15^{\mathrm{a}}$ \\
\hline$>61$ years & $31(52 \%)$ & $25(80 \%)$ & $6(20 \%)$ & \\
\hline \multicolumn{5}{|l|}{ Histological grade } \\
\hline \multicolumn{4}{|l|}{ Tumour stage } & $0.38^{\mathrm{b}}$ \\
\hline pTI & | $(1,7 \%)$ & I (100\%) & & \\
\hline pT2 & $6(10 \%)$ & $4(67 \%)$ & $2(33 \%)$ & \\
\hline pT3 & 47 (80\%) & 35 (75\%) & $12(25 \%)$ & \\
\hline PT4 & $5(8.3 \%)$ & $2(40 \%)$ & $3(60 \%)$ & \\
\hline $\mathrm{pN}$ & & & & $0.78^{\mathrm{a}}$ \\
\hline \multicolumn{4}{|l|}{ Residual tumour } & $0.75^{\mathrm{a}}$ \\
\hline RO & $42(7 \mid \%)$ & 29 (70\%) & $13(30 \%)$ & \\
\hline RI & $17(29 \%)$ & $13(76 \%)$ & $4(24 \%)$ & \\
\hline \multicolumn{4}{|l|}{ UICC stage } & $0.3^{b}$ \\
\hline I & $3(5 \%)$ & | (33\%) & $2(67 \%)$ & \\
\hline$\|$ & $16(27 \%)$ & $13(81 \%)$ & $3(19 \%)$ & \\
\hline III & $25(42 \%)$ & $18(72 \%)$ & $7(28 \%)$ & \\
\hline IV & $12(20 \%)$ & $7(58 \%)$ & $5(42 \%)$ & \\
\hline
\end{tabular}

${ }^{\mathrm{a}}$ Fisher's exact test. ${ }^{\mathrm{b}} \chi^{2}$ test.

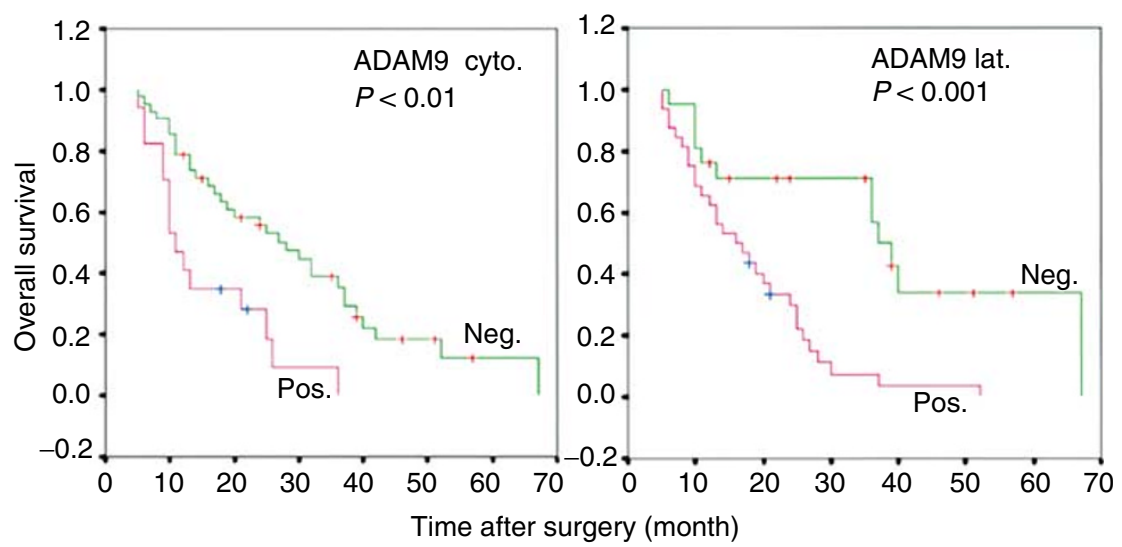

Figure 2 Kaplan-Meier curves of overall survival of PDAC patients showing different ADAM9 expression patterns (cyt: cytoplasmic; lat: basolateral).

independent value of each parameter predicting overall survival in patients with $\mathrm{R} 0$ resection $(N=42)$. Only cytoplasmic expression of ADAM9 and tumour grade were found to be independent prognostic factors for poor overall survival (cytoplasmic ADAM9: $\mathrm{HR}=2.85 ; 95 \% \mathrm{CI}: 1.21-6.71, P<0.05$; tumour grade: $\mathrm{HR}=4.81$; 95\% CI: $2.43-9.52 ; P<0.01$ ) (Table 3).

\section{DISCUSSION}

ADAM9, a member of the ADAM family that is involved in various biological processes (Moss et al, 2001), was found to be overexpressed in prostate carcinoma cell lines, hepatocellular carcinoma and breast carcinoma (McCulloch et al, 2000; Le Pabic et al, 2003; O'Shea et al, 2003). In PDAC, we and others observed ADAM9 overexpression by gene expression profiling. While Iacobuzio-Donahue et al (2003) used cDNA microarray analysis, with subsequent validation of the ADAM9 overexpression by RT PCR in PDAC cell lines, we based our examination on the use of Affymetrix GeneChips and validated the distinct immunohistochemical expression of ADAM9 in a small series of PDACs (Grutzmann et al, 2003).

This study was designed to further validate the significance of ADAM9 overexpression in PDACs by comparing the results with 
Table 3 Results of the univariate and multivariate analyses in patients with pancreatic ductal adenocarcinomas (PDAC)

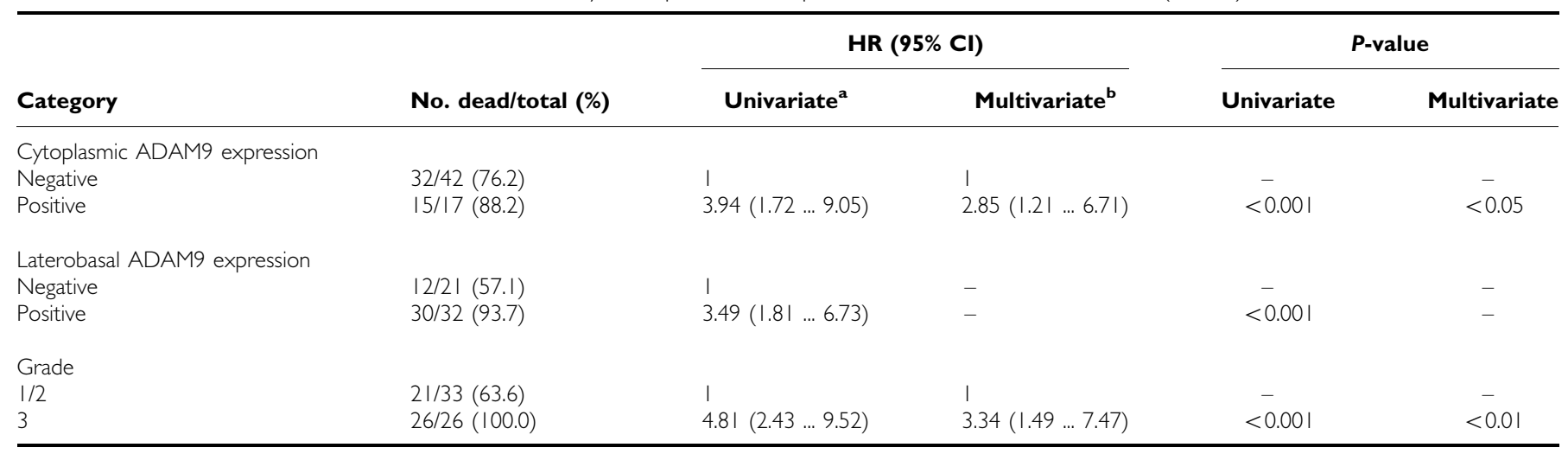

${ }^{\mathrm{a} A}$ total of 59 patients with PDAC. ${ }^{\mathrm{b}} \mathrm{A}$ total of 42 patients with curatively (RO) resected PDAC. HR $=$ hazard ratio.

those obtained in pancreatic tumours other than PDACs, and by correlating the immunohistochemical labelling of the individual PDACs with the survival of the patients.

ADAM9 was detected in 58 of 59 PDACs along the apical lumenoriented membrane of the neoplastic glandular structures. In addition, 32 of the 59 PDACs showed staining of the basolateral cell membrane and 17 revealed cytoplasmic positivity. In the normal adjacent pancreatic tissue, ADAM9 staining, although weak, was also detected at the luminal surface of the interlobular ductal cells and centroacinar cells. It appears that ADAM9 is preferentially a luminal membrane-bound protein of duct-type pancreatic cells. As the apical membrane labelling, observed in the adjacent normal pancreatic tissue, is preserved in almost all PDACs, we might speculate that ADAM9 function could at least partly be maintained in the tumour cells. Among the pancreatic tumours, that is neoplasms of the acinar, endocrine and ductal phenotype, ADAM9 expression was selective for PDACs. Interestingly, endocrine neoplasms did not express ADAM9, although the islet cells in the normal pancreas displayed consistent granular cytoplasmic staining. These results indicate that ADAM9 might not play a role in the biology of nonduct-type neoplasms of the pancreas, but may be important for the biology of PDACs.

The distribution pattern of ADAM9 in PDACs was related to the differentiation of the individual tumours. More than two thirds of the well and moderately differentiated PDACs showed only apical membranous ADAM9 labelling, while poorly differentiated PDACs usually exhibited additional basolateral membranous and cytoplasmic staining. Whether this change bestows a progression advantage on the tumour cells is not yet known. However, because we found that cytoplasmic and basolateral ADAM9 staining correlates with poor survival in PDAC patients, it may be speculated that this over-expression pattern of ADAM9 promotes PDAC progression.

The relationship between the ADAM9 expression pattern in PDAC and the survival probability was tested in a series of curatively resected patients. This test, using a multivariate analysis, revealed cytoplasmic expression of ADAM9 to be an independent prognostic factor in patient survival. The second independent factor detected by this analysis was tumour grade, confirming earlier studies (Luttges et al, 2000). As there was a relationship between differentiation and the ADAM9 expression pattern, the possibility has to be considered that the two factors might be interrelated.

So far the precise function of ADAM9 in the pancreas is unknown. The designation ADAM is derived from their two transmembrane domains, which possess A Disintegrin And a
Metalloprotease function (Izumi et al, 1998). The ADAMs are a multifunctional gene family, some members of which have been shown to play a role in diverse biological processes such as fertilization, myogenesis, neurogenesis and the activation of growth factors/immune regulators such as TNF-alpha. Moreover, ADAM9 is known to cleave heparin-binding EGF-like growth factor (Roghani et al, 1999). The disintegrin function probably relates to cell-to-cell and cell-toextracellular matrix (ECM) adhesive interactions and transduction of signals from the ECM to the cell interior and vice versa. It may be involved in cell migration, invasion, intra- and extravasation and platelet interaction (Poggi et al, 1993; Mizejewski, 1999).

If ADAM9 overexpression is involved in PDAC progression, it may exert its action either via its disintegrin domain or its metalloproteinase domain or, most likely, via both. Various matrix metalloproteinases (MMP) like MMP2 and MMP9 have been described as being overexpressed in PDACs and seem to play an important role in the progression of PDAC (for a review cf. Bloomston et al, 2002). These observations led to a clinical trial of the metalloproteinase inhibitor marimastat in PDAC, which provided evidence of a dose response (Bramhall et al, 2001). Moreover, marimastat is potent not only against MMPs but also against ADAM9 (Moss et al, 2001). It may therefore be speculated that the response to marimastat in patients with PDAC may be in part due to inhibition of ADAM9. If this proved true, ADAM9 might play a role in tumour progression, and might be used not only for prognostic and diagnostic purposes but also for novel therapeutic approaches. Misallocation of ADAM9 from the luminal membrane to the cytoplasm and the basolateral membrane might add to an activation of growth factors and the degradation of ECM by ADAM9.

In conclusion, we have demonstrated that ADAM9 is overexpressed in PDACs but not in endocrine tumours or acinar cell carcinomas. Furthermore, we found a significant association between cytoplasmic ADAM9 overexpression and survival in patients curatively resected for PDAC. This suggests that cytoplasmic ADAM9 overexpression may be a useful diagnostic marker and could also become a potential target in the treatment of PDAC.

\section{ACKNOWLEDGEMENTS}

We thank K Dege for critically reading the manuscript. This paper was supported by Deutsche Krebshilfe (70-2937-SaI). 


\section{REFERENCES}

Amour A, Knight CG, English WR, Webster A, Slocombe PM, Knauper V, Docherty AJ, Becherer JD, Blobel CP, Murphy G (2002) The enzymatic activity of ADAM8 and ADAM9 is not regulated by TIMPs. FEBS Lett 524: $154-158$

Bardeesy N, DePinho RA (2002) Pancreatic cancer biology and genetics. Nat Rev Cancer 2: 897 - 909

Bloomston M, Zervos EE, Rosemurgy II AS (2002) Matrix metalloproteinases and their role in pancreatic cancer: a review of preclinical studies and clinical trials. Ann Surg Oncol 9: 668-674

Bramhall SR, Rosemurgy A, Brown PD, Bowry C, Buckels JA (2001) Marimastat as first-line therapy for patients with unresectable pancreatic cancer: a randomized trial. J Clin Oncol 19: 3447-3455

Grutzmann R, Foerder M, Alldinger I, Staub E, Brummendorf T, Roepcke S, Li X, Kristiansen G, Jesnowski R, Sipos B, Lohr M, Luettges J, Ockert D, Kloeppel G, Saeger HD, Pilarsky C (2003) Gene expression profiles of microdissected pancreatic ductal adenocarcinoma. Virchows Arch 443: $508-517$

Hruban RH, Offerhaus GJ, Kern SE, Goggins M, Wilentz RE, Yeo CJ (1998) Tumor-suppressor genes in pancreatic cancer. J Hepatobiliary Pancreat Surg 5: $383-391$

Iacobuzio-Donahue CA, Maitra A, Olsen M, Lowe AW, Van Heek NT, Rosty C, Walter K, Sato N, Parker A, Ashfaq R, Jaffee E, Ryu B, Jones J, Eshleman JR, Yeo CJ, Cameron JL, Kern SE, Hruban RH, Brown PO, Goggins M (2003) Exploration of global gene expression patterns in pancreatic adenocarcinoma using cDNA microarrays. Am J Pathol 162: $1151-1162$

Izumi Y, Hirata M, Hasuwa H, Iwamoto R, Umata T, Miyado K, Tamai Y, Kurisaki T, Sehara-Fujisawa A, Ohno S, Mekada E (1998) A metalloprotease-disintegrin, MDC9/meltrin-gamma/ADAM9 and PKCdelta are involved in TPA-induced ectodomain shedding of membrane-anchored heparin-binding EGF-like growth factor. EMBO J 17: $7260-7272$

Jemal A, Murray T, Samuels A, Ghafoor A, Ward E, Thun MJ (2003) Cancer statistics, 2003. CA Cancer J Clin 53: 5-26

Kloppel G, Hruban RH, Longnecker DS, Adler G, Kern SE, Partanen TJ (2000) Ductal adenocarcinoma of the pancreas. In: Hamilton SR, Aaltonen LA (eds) Pathology and Genetics of Tumours of the Digestive System. WHO Classification of Tumours. Lyon: IARC Press pp 221-230
Le Pabic H, Bonnier D, Wewer UM, Coutand A, Musso O, Baffet G, Clement B, Theret N (2003) ADAM12 in human liver cancers: TGF-beta-regulated expression in stellate cells is associated with matrix remodeling. Hepatology 37: $1056-1066$

Luttges J, Schemm S, Vogel I, Hedderich J, Kremer B, Kloppel G (2000) The grade of pancreatic ductal carcinoma is an independent prognostic factor and is superior to the immunohistochemical assessment of proliferation. J Pathol 191: $154-161$

McCulloch DR, Harvey M, Herington AC (2000) The expression of the ADAMs proteases in prostate cancer cell lines and their regulation by dihydrotestosterone. Mol Cell Endocrinol 167: 11-21

Mizejewski GJ (1999) Role of integrins in cancer: survey of expression patterns. Proc Soc Exp Biol Med 222: 124-138

Moss ML, White JM, Lambert MH, Andrews RC (2001) TACE and other ADAM proteases as targets for drug discovery. Drug Discov Today 6: $417-426$

O'Shea C, McKie N, Buggy Y, Duggan C, Hill AD, McDermott E, O'Higgins N, Duffy MJ (2003) Expression of ADAM-9 mRNA and protein in human breast cancer. Int J Cancer 105: 754-761

Peto R, Pike MC, Armitage P, Breslow NE, Cox DR, Howard SV, Mantel N, McPherson K, Peto J, Smith PG (1977) Design and analysis of randomized clinical trials requiring prolonged observation of each patient. II. Analysis and examples. $\mathrm{Br}$ J Cancer 35: $1-39$

Poggi A, Stella M, Donati MB (1993) The importance of blood cell-vessel wall interactions in tumour metastasis. Baillieres Clin Haematol 6: $731-752$

Roghani M, Becherer JD, Moss ML, Atherton RE, Erdjument-Bromage H, Arribas J, Blackburn RK, Weskamp G, Tempst P, Blobel CP (1999) Metalloprotease-disintegrin MDC9: intracellular maturation and catalytic activity. J Biol Chem 274: $3531-3540$

Slebos RJ, Hoppin JA, Tolbert PE, Holly EA, Brock JW, Zhang RH, Bracci PM, Foley J, Stockton P, McGregor LM, Flake GP, Taylor JA (2000) K-ras and p53 in pancreatic cancer: association with medical history, histopathology, and environmental exposures in a population-based study. Cancer Epidemiol Biomarkers Prev 9: $1223-1232$ 